IN BRIEF

\section{$\Rightarrow$ HEADACHE}

\section{Complete withdrawal more feasible and effective than restriction in medication-overuse headache}

Completely stopping medication is both more feasible and more effective than restricting medication intake for the treatment of medication-overuse headache $(\mathrm{MOH})$, according to a new study. Engelstoft and co-workers randomly assigned patients with $\mathrm{MOH}$ to either 2 months of medication withdrawal without any analgesics or acute migraine medications (complete withdrawal group) or to continuation of usual analgesics and/or migraine medication up to 2 days per week during the withdrawal period (restricted intake group). Patient-graded feasibility of withdrawal was significantly higher in the complete withdrawal group than in the restricted intake group. At 12 months, psychological dependence on headache medication showed greater reductions in the complete withdrawal group than in the restricted intake group; headache-related anxiety was also reduced to a greater extent in the complete withdrawal group.

ORIGINAL ARTICLE Engelstoft, I. M. et al. Complete withdrawal is the most feasible treatment for medication-overuse headache: a randomized controlled open-label trial. Eur.J. Pain https://doi.org/10.1002/ejp.1383 (2019)

\section{$\Rightarrow$ ALZHEIMER DISEASE}

\section{New blood-based biomarkers for Alzheimer disease}

A predictive model combining age with plasma arginine and C5 acylcarnitine levels shows promise for identifying Alzheimer disease (AD) in older adults, say researchers. Individuals included in the study were aged 55-90 years and were classified as being healthy controls $(n=15)$ or having mild cognitive impairment $(n=10)$ or $\mathrm{AD}(n=15)$. The researchers found that plasma levels of several metabolites differed significantly between controls and patients in early or progressive stages of $\mathrm{AD}$. Age and arginine and $\mathrm{C} 5$ acylcarnitine concentrations were the best discriminative variables for AD classification and were used to create a predictive model that could distinguish patients with $\mathrm{AD}$ from the other groups with $60 \%$ sensitivity and $86.7 \%$ specificity. However, in the mild cognitive impairment group, only $37.5 \%$ of patients were classified correctly, indicating that the model has limited diagnostic value for this condition.

ORIGINAL ARTICLE Lin, C.-N. et al. A metabolomic approach to identifying biomarkers in blood of Alzheimer's disease. Ann. Clin. Transl Neurol. https://doi.org/10.1002/acn3.726 (2019)

\section{STROKE}

\section{Genetic variant associated with stroke outcomes}

Patient outcomes after ischaemic stroke vary widely, and a new genome-wide association study (GWAS) reports a genetic variant associated with outcome 3 months after stroke. The GWAS by Söderholm et al. involved 6,165 patients from 12 studies of ischaemic stroke. The intronic variant rs 1842681 in LOC105372028 - a gene involved in the expression of protein phosphatase 1 , which is implicated in brain plasticity - had a significant association with functional outcomes 60-190 days after stroke. Several other variants, some within or near genes that have been linked to outcomes in animal models of stroke, showed a suggestive association with patient outcomes. The researchers say that larger studies are needed to confirm the finding and explore the potential functional mechanisms.

ORIGINAL ARTICLE Söderholm, M. et al. Genome-wide association meta-analysis of functional outcome after ischemic stroke. Neurology https://doi.org/10.1212/ WNL.0000000000007138 (2019)

\title{
Neurofilament makes light of MS treatment monitoring
}

Serum levels of neurofilament light chain (NfL) can be used to monitor treatment responses and safety in multiple sclerosis (MS), according to two new studies. The findings indicate that NfL could be used to identify patients with a poor response or who are developing serious adverse effects.

$\mathrm{NfL}$ is a neuronal structural protein that is released following neuroaxonal damage, and previous work has demonstrated that NfL is a promising biomarker of MS disease activity and progression. Jens Kuhle and colleagues have now taken this work one step further.

"Several studies have shown that NfL is increased in cerebrospinal fluid and blood as a function of relapse activity of MS," explains Kuhle. "Whether NfL levels would decrease under therapy was an obvious next question."

To address this question, Kuhle and colleagues analysed NfL levels in serum samples from 589 participants in two phase III clinical trials of fingolimod in relapsing-remitting MS: the placebo-controlled FREEDOMS trial and the IFN $\beta 1 \mathrm{a}-$ controlled TRANSFORMS trial. Serum samples were collected at baseline and at 6,12, 18 and 24 months in FREEDOMS, and at baseline and 12 months in TRANSFORMS. NfL levels were measured with the single molecule array (SIMOA) immunoassay.

As previously shown, higher baseline serum levels of NfL were associated with greater disease activity. However, the new analysis also demonstrated that NfL levels were significantly lower at all time points in patients who received fingolimod than in patients who received placebo or IFN $\beta 1$. By the end of the studies, levels approached those in healthy controls.

"We have, for the first time, a biofluid marker at hand that measures drug response in MS," says Kuhle. "The icing on the cake is that the clinical experience that fingolimod is more potent than IFN $\beta 1 \mathrm{a}$ is reflected by the degree of decrease in NfL."

Nevertheless, more work needs to be done to develop NfL as a biomarker for use in individual patients. "Our next endeavour is to establish age-related normal values," Kuhle says. "Standardization and refinement of the assay platform is another aspect."

In the second new study, serum NfL levels were investigated as a marker of progressive multifocal leukoencephalopathy (PML), a rare but serious adverse effect of natalizumab treatment. Led by Giancarlo Comi, the researchers analysed serum NfL levels in 161 natalizumab-treated patients with MS, 25 of whom had PML, and 151 untreated patients with MS.

Onset of PML was associated with a tenfold increase in serum levels of NfL. Furthermore, among patients receiving natalizumab, serum levels of NfL could be used to discriminate between patients who were developing PML and those who were not, and between patients who were developing PML and those who were experiencing an MS relapse.

"If validated in larger cohorts of patients, serum levels of NfL may be used as a biomarker to monitor not only drug efficacy, but also the occurrence and early recognition of PML," the authors conclude in the paper.

ORIGINAL ARTICLES Kuhle, J. et al. Blood neurofilament light chain as a biomarker of MS disease activity and treatment response. Neurology https://doi.org/10.1212/ WNL.0000000000007032 (2019)| Dalla Costa, G. et al. Serum neurofilaments increase at PML onse in natalizumab-treated MS patients. Ann. Neurol. https://doi.org/10.1002/ana.25437 (2019) FURTHER READING Khalil, M. et al. Neurofilaments as biomarkers in neurological disorders. Nat. Rev. Neurol. 14, 577-589 (2018) 